\title{
Investigation of point defect generation in dry etched InP ridge waveguide structures
}

\author{
M. Avella and J. Jiménez ${ }^{a}$ \\ Física Materia Condensada, ETSII, 47011 Valladolid, Spain \\ F. Pommereau \\ Alcatel-Thales III-V Laboratory, 91128 Palaiseau, France
}

\author{
J. P. Landesman and A. Rhallabi \\ Laboratoire des Plasmas et des Couches Minces, Institut des Matériaux, UMR6502 CNRS, 2, \\ Rue de la Houssinière, 44322 Nantes cedex 3, France
}

(Received 26 November 2006; accepted 3 May 2007; published online 1 June 2007)

\begin{abstract}
Waveguides engraved in InP by dry etching, reactive ion etching and inductively coupled plasma (ICP), were studied by cathodoluminescence. The dry etching processes were found to induce nonradiative recombination centers, which reduce the luminescence emission from the ridge structures. In addition, the ICP process introduced intrinsic defects, probably In vacancy related defects, which were generated at the dielectric cap/InP interface at the ridge top. (C) 2007 American Institute of Physics. [DOI: 10.1063/1.2743384]
\end{abstract}

Ion etching techniques are used for the fabrication of III-V semiconductor structures with submicrometric lateral dimension. ${ }^{1}$ Therefore, they are basic tools for the fabrication of optoelectronic devices, such as vertical cavity surface emitting lasers, buried heterostructure lasers, and photonic crystals for integrated optical devices. ${ }^{2}$ In this type of devices, one aims for vertical sidewalls and smooth surfaces, because rough etched surfaces lead to enhanced surface recombination velocity (SRV), light scattering, and optical propagation losses that affect the device performance and reliability. Therefore, most of the work concerning the characterization of selective area etched structures is based on the morphology analysis, e.g., the aspect ratio and the verticality of the etched sidewalls, and the surface damage. ${ }^{3,4}$ In the last few years, inductively coupled plasma (ICP) has had more applications, since it allows low ion energy $(<100 \mathrm{eV})$ with higher etching rate than reactive ion etching (RIE) does while maintaining smooth surfaces.

One of the collateral effects of the ion etching is the formation of defects. The study of these effects has mainly concerned the etched surfaces, with special emphasis paid to the etched sidewalls. However, as important as the etched surfaces are the micrometric engraved structures, which constitute the active parts of the devices and have not received enough attention. The generation of point defects in these structures has been normally indirectly measured by studying quantum well intermixing enhanced by the diffusion of point defects under thermal annealing. ${ }^{5,6}$ However, a complete view of where the defects are formed during the etching step is still lacking. The study of small structures should require the use of spectroscopic techniques with submicrometric spatial resolution. In particular, cathodoluminescence (CL) in the scanning electron microscope (SEM) appears as a unique tool to study such structures because of its high spatial resolution, its coupling to the morphology observation in the SEM, and its spectroscopic capabilities.
The CL measurements were carried out at $80 \mathrm{~K}$ in a $\mathrm{XiCLOne} \mathrm{system} \mathrm{from} \mathrm{Gatan.} \mathrm{The} \mathrm{detection} \mathrm{is} \mathrm{carried} \mathrm{out} \mathrm{by}$ means of a charge coupled device camera allowing the acquisition of the full spectrum for each e-beam scanned pixel. Etched structures were studied in both top view and cross section on cleaved surfaces.

The samples were fabricated on bulk (100) InP S doped at $1.5 \times 10^{18} \mathrm{~cm}^{-3}$. A $400 \mathrm{~nm}$ silicon nitride layer was deposited by plasma enhanced chemical vapor deposition at $250{ }^{\circ} \mathrm{C}$. Ridge patterns with various widths ranging from 1 to $3 \mu \mathrm{m}$ were defined in a positive photoresist by contact lithography and transferred into $\mathrm{SiN}_{x}$ by RIE using a $\mathrm{SF}_{6} / \mathrm{CHF}_{3} / \mathrm{O}_{2}$ gas mixture. After resist and polymer stripping in an $\mathrm{O}_{2}$ microwave plasma asher, the ridge stripes have been transferred into InP by RIE or ICP etching.

The RIE was carried out on an Adixen GIR100 equipment using a mixture of $\mathrm{CH}_{4} / \mathrm{H}_{2}$ gases. The ion energy at the surface of the sample was as high as $470 \mathrm{~V}$. This was the value of the voltage at the cathode. The beam current density was estimated at $3 \mathrm{~mA} / \mathrm{cm}^{2}$. Hydrocarbon polymers which formed along the sidewall of the ridges and on the SiN mask have been removed by $\mathrm{O}_{2}$ microwave plasma etching.

The ICP etching was performed in an Adixen 601E equipment using $\mathrm{SiCl}_{4}$ based chemistry. The plasma is excited with a $13.56 \mathrm{MHz}$ radio frequency (rf) generator and

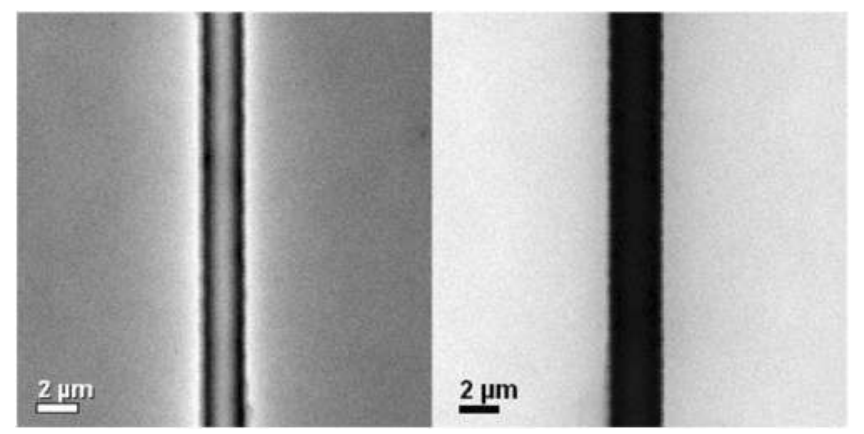

FIG. 1. Panchromatic CL images of RIE (left) and ICP (right) ridges in top view.

${ }^{a)}$ Electronic mail: jimenez@fmc.uva.es 


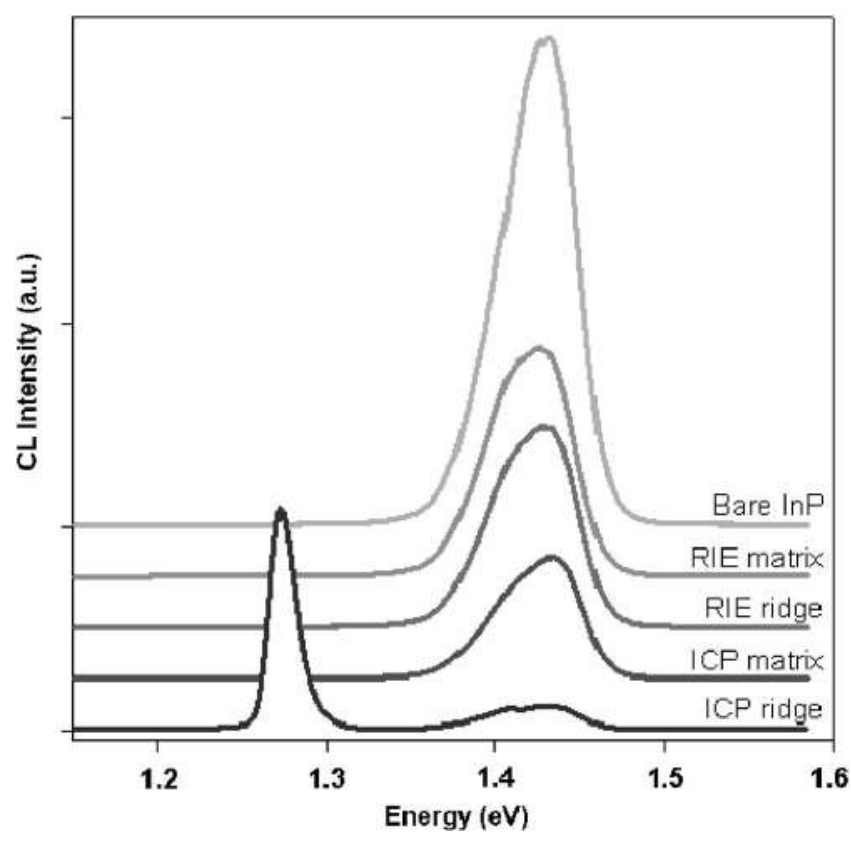

FIG. 2. CL spectra in different samples and positions (see insert legend).

the sample is biased with a separate rf generator at 13.56 MHz. The resulting ion energy was optimized at $60 \mathrm{~V}$ and the ion beam current was estimated at $8 \mathrm{~mA} / \mathrm{cm}^{2}$. For both RIE and ICP treated samples the $\mathrm{Si}_{3} \mathrm{~N}_{4}$ mask was removed with fluorhydric acid. The ridge heights are 3.6 and $4 \mu \mathrm{m}$ for RIE and ICP samples, respectively. For more details about the experimental procedure, see Ref. 7 .

The CL images show a strong contrast between the ridge (nonetched) and the etched floor for both ICP and RIE processes (Fig. 1). Surprisingly, the ridge displays a lower luminescence intensity giving a dark contrast in the CL image, while the etched floor appears bright, contrary to the expected behavior not only because the SRV of the etched regions must be enhanced due to surface damage but also because the ion bombardment is expected to create point defects. ${ }^{3}$ The low luminescence emission in the ridges can be due to the influence of the etched sidewalls, and/or the generation of nonradiative recombination centers.

Local spectra were acquired in order to check the differences between the two processes and the two regions, etched floor and nonetched ridges. The spectrum in the etched floor presents a broad near band gap (NBG) band composed of at least two bands, corresponding to the band to band recombination $(1.43 \mathrm{eV})$ and a shoulder peaking after deconvolution at $1.395 \mathrm{eV}$, which is usually associated with a free to bound transition. This is a typical spectrum of S-doped InP in the mid- $10^{18}-\mathrm{cm}^{-3}$ doping level. The peak energies are shifted to the blue due to conduction band filling (Burstein-Moss effect). ${ }^{8}$ This spectrum is common to both processes and is similar to the reference substrate (Fig. 2). What is different between the two etching processes is the spectrum inside the ridge. While the spectrum corresponding to the RIE process is similar to that observed in the etched region with the corresponding intensity decrease, the spectrum in the ridge of the sample etched by ICP presents an additional band, peaking around $1.27 \mathrm{eV}$, the intensity of which can be as high as the one of the NBG band. This band evidences the generation of bulk point defects during the ICP process in the regions that are not directly exposed to the ICP ion bombardment. The influence of a dielectric cap layer in the formation and diffusion of defects has been discussed in the case of InP and related alloys $;, 10$ however, temperatures much higher than the nominal temperature of the process (the samples were thermalized at $20{ }^{\circ} \mathrm{C}$ ) are necessary to generate defects at the $\mathrm{InP} / \mathrm{SiN}_{x}$ interface by the only thermal energy transfer. Because the etched regions do not show additional luminescence bands related to defects, one can argue that the ICP process itself does not create the point defects in regions that are not capped with the dielectric layer. Therefore, the $1.27 \mathrm{eV}$ band must arise from defects created during the ICP etching of micrometer structures by the presence of the dielectric capping layer. This luminescence band in InP is usually associated with In vacancies or their complexes. ${ }^{11}$.

Ridges of different dimensions, 1 and $2 \mu \mathrm{m}$ wide, respectively, were studied in cross section. The distribution of defects and the influence of the surface are seen in Fig. 3, where monochromatic images obtained at $1.43 \mathrm{eV}(866 \mathrm{~nm})$ and $1.27 \mathrm{eV}(975 \mathrm{~nm})$ for the ICP and RIE samples are shown. The images obtained at $1.43 \mathrm{eV}$ show the recombination pattern associated with the increase of the SRV by the etching processes at both the sidewalls and the etched floor and the nonradiative recombination activity of the $\mathrm{InP} / \mathrm{SiN}_{x}$ interface on top of the ridge, where the luminescence intensity is drastically reduced. The luminescence inside the ridges is significantly reduced in the $1 \mu \mathrm{m}$ wide ridge with respect to the $2 \mu \mathrm{m}$ wide ridge, which is accounted for by the nonradiative recombination at the etched sidewalls, which have more relevance in narrow ridges [see Fig. 3(d)]. The $1.27 \mathrm{eV}$ band in ICP etched structures is very intense on top of the ridge [Figs. 3(c) and 3(e)]; the highest intensity of the $1.27 \mathrm{eV}$ band is found beneath the dielectric capping layer around $1 \mu \mathrm{m}$ away from the interface; the nonradiative recombination centers are dominant in the region close to the interface. The distribution of this luminescence band shows that the point defects are generated at the $\mathrm{InP} / \mathrm{SiN}_{x}$ interface and thereafter they in-diffuse through the ridge expanding beneath the ridge when they reach the ridge base [see Figs. 3(c) and 3(e)], where the amplitude of the $1.27 \mathrm{eV}$ band is mapped in the two ICP etched ridges, 2 and $1 \mu \mathrm{m}$ wide,

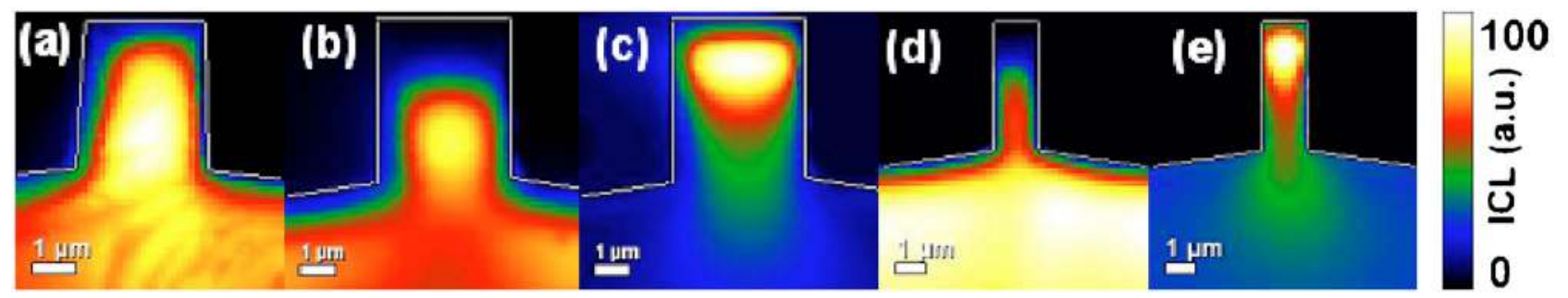


respectively. This distribution pattern evidences that the defects responsible for the $1.27 \mathrm{eV}$ band are not generated at the sidewalls, nor at the etched floor. Therefore they are not a direct product of the ion bombardment. During ion bombardment the energy transfer from the incident ions, which lose the energy in cascade collisions, to the InP lattice can lower the potential barrier for defect formation and motion or can transfer heat to the lattice enhancing the temperature. The energy of the ions is higher in RIE than in ICP; however, the etching rate is 25 times faster in ICP with respect to RIE (500 against $20 \mathrm{~nm} / \mathrm{min}$ ), which suggests that the high ion flow and the nature of the etching gases are prevalent in the formation of the In vacancy related defects responsible for the $1.27 \mathrm{eV}$ luminescence band. In this process, there is a higher transfer of energy to the lattice allowing the formation of the vacancy defects in the ICP process. It should be noted that the formation of those defects must be strongly dependent on the characteristics of the dielectric capping layer. The dielectric capping layer plays the role of a defect source during the ICP process. Therefore, the nature and stoichiometry of the dielectric are relevant to the formation of defects. ${ }^{12}$

On the other hand, the diffusionlike distribution of the $1.27 \mathrm{eV}$ band [Figs. 3(c) and 3(e)], suggests that the temperature inside the ridge structures is enhanced during the etching process. The heat dissipation must be slowed due to the reduced dimension of the ridge structures; see that the narrow ridge [Fig. 3(e)] shows a longer diffusion range than the wider ridge [Fig. 3(c)]. Further annealing experiments are necessary to elucidate the temperature enhancement inside the ridge structures during dry etching.

In summary, we have demonstrated that point defects are generated during the fabrication of ridgelike structures in InP by ICP dry etching processes, but not by the RIE process. Indium vacancy related defects were observed in ICP etching, which are responsible for an additional luminescence band at $1.27 \mathrm{eV}$. The generation of these point defects is located at the $\mathrm{InP} / \mathrm{SiN}_{x}$ interface and were demonstrated to in-diffuse toward the base of the ridge during the etching process.

This work was carried out in the frame of the SpanishFrench Cooperation Program (Picasso Integrated Actions).

${ }^{1}$ S. J. Choi, K. Djorjev, S. J. Choi, and P. D. Dapkus, J. Vac. Sci. Technol. B 20, 301 (2002).

${ }^{2}$ Ch. Kim, W. J. Kim, A. Stapleton, J. R. Cao, J. D. O'Brien, and P. D. Dapkus, J. Opt. Soc. Am. B 19, 1777 (2002).

${ }^{3}$ H. Iber, S. Mo, E. Peiner, G. Vollrath, A. Schlachetzki, and F. Fiedler, Semicond. Sci. Technol. 12, 755 (1997).

${ }^{4}$ S. J. Pearton, F. Ren, W. S. Hobson, C. A. Green, and U. K. Chakrabarti, Semicond. Sci. Technol. 7, 1217 (1992).

${ }^{5}$ B. S. Ooi, A. C. Bryce, C. D. W. Wilkinson, and J. H. Marsh, Appl. Phys. Lett. 64, 598 (1994).

${ }^{6}$ Ching-Hui Chen, D. Ginger Yu, E. L. Hu, and P. M. Petroff, J. Vac. Sci. Technol. B 14, 3684 (1996).

${ }^{7}$ F. Pommereau, L. Legouezigou, S. Hubert, S. Sainson, J. P. Chandouineau, S. Fabre, G. H. Duan, B. Lombardet, R. Ferrini, and R. Houdré, J. Appl. Phys. 95, 2242 (2004).

${ }^{8}$ M. Bugaajski and W. Lewandowski, J. Appl. Phys. 57, 521 (1985).

${ }^{9}$ S. Sudo, H. Onishi, Y. Nakano, Y. Shimogaki, K. Tada, M. J. Mondry, and L. A. Coldren, Jpn. J. Appl. Phys., Part 1 35, 1276 (1996).

${ }^{10}$ W. J. Choi, H. T. Yi, J. I. Lee, and D. H. Woo, J. Korean Phys. Soc. 45, 773 (2004).

${ }^{11}$ H. Temkin, B. V. Dutt, W. A. Bonner, and V. G. Keramidas, J. Appl. Phys. 53, 7526 (1982).

${ }^{12}$ J. S. Yu, J. D. Song, Y. T. Lee, and H. Lim, J. Appl. Phys. 92, 1386 (2002). 